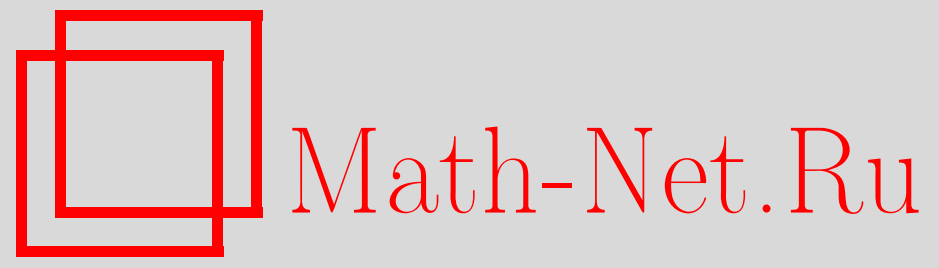

А. В. Чашкин, Среднее время вычисления значений элементарных булевых функций, Дискрет. матем., 2000, том 12, выпуск 4, 109-120

DOI: https://doi.org/10.4213/dm348

Использование Общероссийского математического портала Math-Net.Ru подразумевает, что вы прочитали и согласны с пользовательским соглашением http://www . mathnet.ru/rus/agreement

Параметры загрузки:

IP : 54.162 .85 .209

26 апреля 2023 г., 07:30:13

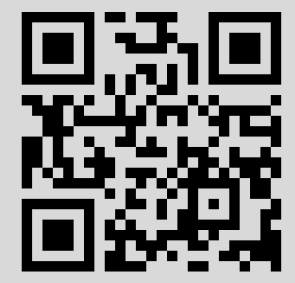


УДК 519.7

\title{
Среднее время вычисления значений элементарных булевых функций
}

\author{
(c) 2000 г. A. B. Чашкин
}

\begin{abstract}
Рассматривается реализация дизъюнкции, конъюнкции и линейной функции, зависящих от растущего числа аргументов, неветвящимися программами с условной остановкой. Найдены асимптотически точные формулы для среднего времени вычисления значений этих функций. Установлено, что средние времена вычисления дизъюнкции и конъюнкции - постоянные величины, а среднее время вычисления линейной функции совпадает со сложностью реализации этой функции схемами из функциональных элементов.

Работа выполнена при поддержке Российского фонда фундаментальных исследований, проект 99-01-01175, и ФЦП «Интеграция», проект 473.
\end{abstract}

\section{1. Введение}

В работе изучается среднее время вычисления значений простейших булевых функций неветвящимися программами с условной остановкой. Рассматриваемые ниже программы обобщают понятие схемы из функциональных элементов [1] и являются естественной моделью неветвящихся вычислений, то есть вычислений, в которых нет условного перехода и косвенной адресации, но есть возможность досрочного прекращения работы при выполнении определенного условия. Такие вычисления можно представить следующим образом. Вычисления выполняет процессор, снабженный памятью, состоящей из отдельных ячеек. Процессор способен вычислять некоторое количество двуместных элементарных функций; множество этих функций назовем базисом вычисления. Каждая ячейка памяти в любой момент времени доступна процессору как для чтения, так и для записи информации. Процессор работает под управление программы, являющейся последовательностью элементарных операторов двух видов. Каждый оператор первого вида вычисляет значение некоторой базисной функции, аргументами которой является содержимое определенных ячеек памяти. Вычисленный результат также помещается в одну из ячеек памяти. Оператор второго вида может прекратить выполнение программы. Каждый такой оператор имеет единственный аргумент - содержимое некоторой ячейки памяти. Если значение аргумента равно определенному фиксированному числу, например, единице, то процессор прекращает работу. Если значение аргумента иное, то выполняется следующий оператор программы. В памяти выделяется особая ячейка, содержимое которой после прекращения работы объявляется результатом работы программы. Естественной мерой сложности таких программ является среднее по 
всем возможным аргументам время работы. Изучение этой меры сложности представляет не меньший интерес, чем изучение сложности реализации функций схемами из функциональных элементов, определяющей время вычисления в худшем случае.

Ранее модели, соответствующие неветвящимся вычислениям с условной остановкой, были рассмотрены в [2-6]. В этих работах были найдены оценки функций Шеннона среднего времени вычисления булевых операторов, булевых функций из некоторых специальных классов, установлен ряд общих свойств неветвящихся программ. Конкретные булевы функции в [2-6] не изучались. В настоящей работе изучается среднее время вычисления значений простейших булевых функций: дизъюнкции, конъюнкции и линейной функции. Для дизъюнкции и конъюнкции растущего числа аргументов найдены асимптотически точные значения среднего времени вычисления их значений, эти значения - постоянные величины. Для среднего времени вычисления значений линейной функции найдена точная формула. Показано, что при вычислении линейной функции возможность досрочного прекращения вычислений не позволяет уменьшить число выполняемых операций, то есть среднее время вычисления линейной функции совпадает со сложностью реализации этой функции схемами из функциональных элементов.

\section{2. Определения}

Дадим определения основных понятий, используемых в работе.

Пусть $P_{2}^{2}-$ множество всех двуместных булевых функций, $X=\left\{x_{1}, \ldots, x_{n}\right\}$ - множество независимых переменных, $Y=\left\{Y_{1}, \ldots, Y_{l}\right\}-$ множество внутренних переменных, $Z$ - выходная переменная.

Пусть $A \in Y \cup\{Z\}, B, C \in X \cup Y \cup\{Z\}, f \in P_{2}^{2}$. Выражение

$$
p: \quad A=f(B, C)
$$

назовем функциональным оператором. Величину $A$ назовем выходом функционального оператора $p$, а величины $B, C$ назовем входами этого оператора. Пусть, далее, $A \in X \cup Y \cup\{Z\}$. Выражение

$$
p: \quad \operatorname{Stop}(A)
$$

назовем оператором остановки. Величину $A$ назовем входом оператора остановки $p$.

Пусть $P=p_{1} \ldots p_{i} \ldots p_{L}$ - последовательность операторов. Последовательность $P$ назовем неветвящейся программой с условной остановкой, если каждый вход оператора $p_{j}, j \in\{1,2, \ldots, L\}$, есть либо входная переменная, либо выход некоторого функционального оператора $p_{i}$ при $i<j$.

Неветвящаяся программа работает в дискретные моменты времени $t=0,1, \ldots$ Индуктивно определим значения внутренних $Y_{i}(x ; t)$ и выходной $Z(x ; t)$ переменных программы $P$ в произвольный момент времени $t$ на наборе независимых переменных $x=\left(x_{1}, \ldots, x_{n}\right)$ :

- в начальный момент времени $t=0$, значения всех внутренних и выходной переменных считаем неопределенными;

- если внутренняя переменная $Y_{j}$ (выходная переменная $Z$ ) не является выходом оператора $p_{t}$, то полагаем

$$
Y_{j}(x ; t)=Y_{j}(x ; t-1), \quad Z(x ; t)=Z(x ; t-1) ;
$$


- если внутренняя переменная $Y_{j}$ (выходная переменная $Z$ ) является выходом оператора $p_{t}$ и $B(x ; t-1), C(x ; t-1)$ - значения входов оператора $p_{t}$ в момент времени $t-1$, то полагаем

$$
\begin{aligned}
Y_{j}(x ; t) & =f_{t}(B(x ; t-1), C(x ; t-1)), \\
Z(x ; t) & =f_{t}(B(x ; t-1), C(x ; t-1)) .
\end{aligned}
$$

Значением оператора $p_{t}$ на наборе независимых переменных $x=\left(x_{1}, \ldots, x_{n}\right)$ назовем значение его выхода в момент времени $t$ и обозначим через $p_{t}(x)$.

Пусть $n(p)$ - номер оператора $p$ в программе $P$, то есть $n\left(p_{i}\right)=i$. Пусть $p_{t_{1}}, \ldots, p_{t_{r}}$ - все операторы остановки из $P, t_{1}<\ldots<t_{r}$. Через $s_{j}$ обозначим $j$-й оператор остановки программы $P$, то есть $s_{j}=p_{t_{j}}$, а через $q_{j}$ обозначим единственный аргумент этого оператора.

Будем говорить, что $k$-й оператор остановки $s_{k}$ останавливает вычисления программы $P$ на наборе $x$, если

$$
q_{1}(x)=\ldots=q_{k-1}(x)=0, \quad q_{k}(x)=1 .
$$

Результат действия программы $P$ на наборе $x$ обозначим через $P(x)$ и его $j$-ю компоненту $P_{j}(x)$ определим следующим образом:

$$
P_{j}(x)= \begin{cases}Z_{j}\left(x ; t_{k}\right), & \text { если } q_{1}(x)=\ldots=q_{k-1}(x)=0, q_{k}(x)=1, \\ Z_{j}(x ; L), & \text { если } q_{1}(x)=\ldots=q_{k}(x)=0,\end{cases}
$$

то есть $P_{j}(x)$ равно значению $j$-й выходной переменной в момент остановки программы. Легко видеть, что

$$
\begin{aligned}
P_{j}(x) & =q_{1}(x) Z_{j}\left(x ; t_{1}\right) \vee \ldots \vee \bar{q}_{1}(x) \bar{q}_{2}(x) \ldots \bar{q}_{k-1}(x) q_{k}(x) Z_{j}\left(x ; t_{k}\right) \vee \ldots \\
& \vee \bar{q}_{1}(x) \bar{q}_{2}(x) \ldots \bar{q}_{r-1}(x) q_{r}(x) Z_{j}\left(x ; t_{r}\right) \vee \bar{q}_{1}(x) \bar{q}_{2}(x) \ldots \bar{q}_{r}(x) Z_{j}(x ; L) .
\end{aligned}
$$

Сложностью $L(P)$ программы $P$ назовем число операторов этой программы. Временем работы $T_{P}(x)$ программы $P$ на наборе переменных $x$ назовем минимальное $n\left(s_{j}\right)$ такое, что $q_{j}(x)=1$, то есть это число операторов, выполненных до остановки программы. Если все $q_{j}(x)=0$, то выполняются все операторы программы и в этом случае $T_{P}(x)=L(P)$. Величину

$$
T(P)=2^{-n} \sum T_{P}(x)
$$

где суммирование производится по всем двоичным наборам длины $n$, назовем средним временем работы программы $P$. Если для некоторой булевой функции $f$ и любого двоичного набора $x$ справедливо равенство $f(x)=P(x)$, то будем говорить, что программа $P$ вычисляет функцию $f$. Величину

$$
T(f)=\min T(P),
$$

где минимум берется по всем программам, вычисляющим $f$, назовем средним временем вычисления функции $f$. Программу $P$, вычисляющую функцию $f$, для которой справедливо равенство $T(P)=T(f)$, назовем минимальной программой. Число элементов минимальной схемы из функциональных элементов, реализующей функцию $f$ в базисе из всех двуместных булевых функций, назовем сложностью $f$ и обозначим символом $L(f)$.

Отметим, что неветвящаяся программа без операторов остановки является обычной схемой из функциональных элементов. 


\section{3. Приведенные программы}

Перед доказательством основных результатов введем понятие приведенной программы и покажем, что любая программа без увеличения сложности и среднего времени работы может быть преобразована в приведенную программу.

Пусть $A \in X \cup Y \cup\{Z\}$ и $A$ является входом (выходом) оператора $p_{t}$ программы $P$. Будем говорить, что на входе (на выходе) оператора $p_{t}$ вычисляется функция $f(x)$, если $A(x ; t-1)=f(x)(A(x ; t)=f(x))$ при всех значениях независимых переменных, при которых значение переменной $A$ определено.

Пусть $A \in X \cup Y \cup\{Z\}$ и $A$ является входом операторов $p_{i}$ и $p_{j}$ программы $P$. Будем говорить, что два оператора $p_{i}$ и $p_{j}$ имеют общий вход, если $A(x ; i-1)=A(x ; j-1)$ при всех значениях независимых переменных, при которых значение переменной $A$ определено.

Программу $P$ назовем приведенной, если вход каждого оператора программы $P$ не является тождественной постоянной и никакие два оператора остановки программы $P$ не имеют общего входа.

Лемма 1. Произвольная программа $P$ может быть преобразована в приведенную программу $P^{\prime} \operatorname{max,~что~}$

$$
\begin{gathered}
T\left(P^{\prime}\right) \leqslant T(P), \quad L\left(P^{\prime}\right) \leqslant L(P), \\
P^{\prime}\left(x_{1}, \ldots, x_{n}\right)=P\left(x_{1}, \ldots, x_{n}\right)
\end{gathered}
$$

при всех $\left(x_{1}, \ldots, x_{n}\right)$.

Доказателъство. Пусть $P$ - произвольная программа, $x=\left(x_{1}, \ldots, x_{n}\right)$ - набор независимых переменных, $p_{t}: A=g(B, C)$ - оператор, на первом входе которого вычисляется постоянная функция, то есть $B(x ; t-1)$ есть постоянная. В этом случае $A(x ; t)$ зависит только от $C(x ; t-1)$, и, следовательно, существует функция $h$ такая, что

$$
h(C(x ; t-1))=g(B(x ; t-1), C(x ; t-1)) .
$$

Заменяя в $P$ оператор $p_{t}$ оператором $p_{t}^{\prime}: A=h(C)$, получаем программу, удовлетворяющую условиям леммы.

Допустим теперь, что в программе $P$ найдутся два оператора остановки $p_{i}$ и $p_{j}$, $i<j$, с одним и тем же входом $A$. Если $A(x ; i-1)=A(x ; j-1)=1$, то оператор $p_{i}$ останавливает выполнение программы и $p_{j}$ не выполняется. Если $A(x ; j-1)=0$, то оператор $p_{j}$ не останавливает выполнение программы. Следовательно, оператор $p_{j}$ может быть удален из программы $P$ и это никак не скажется на ее работе. Лемма доказана.

Далее рассматриваем только приведенные программы.

\section{4. Результаты}

Теорема 1. $\Pi p u n \rightarrow \infty$

$$
T\left(x_{1} \vee x_{2} \vee \ldots \vee x_{n}\right) \sim \frac{8}{3}
$$


Доказателъство. Верхнюю оценку среднего времени получим, оценив среднее время работы приводимой ниже программы $P_{\vee}$, вычисляющей дизъюнкцию $n$ переменных. Без ограничения общности полагаем, что $n-$ четное число.

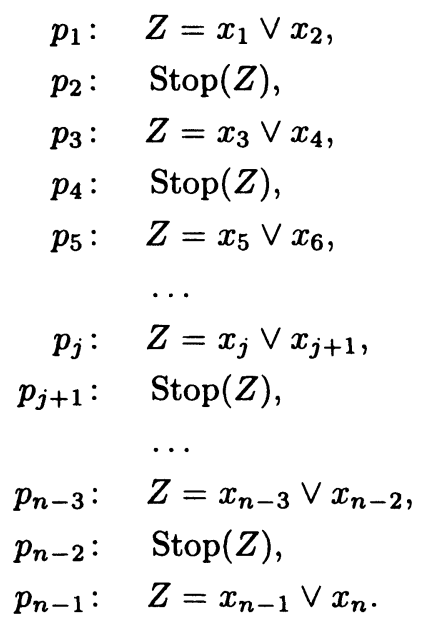

Легко видеть, что

$$
\begin{aligned}
T\left(P_{\vee}\right) & \leqslant \frac{1}{2^{n}} \sum_{j=1}^{n / 2} 2 j \cdot 3 \cdot 2^{n-2 j}<6 \sum_{j=1}^{\infty} \frac{j}{4^{j}} \\
& =6 \sum_{j=1}^{\infty} \sum_{i=j}^{\infty} \frac{1}{4^{i}}=6 \sum_{j=1}^{\infty} \frac{1}{4^{j}} \frac{1}{1-1 / 4}=6 \cdot \frac{4}{3} \cdot \frac{1}{4} \cdot \frac{4}{3}=\frac{8}{3} .
\end{aligned}
$$

Найдем теперь нижнюю оценку для дизъюнкции растущего числа аргументов. Отметим, что минимальная программа $P_{n}$, вычисляющая дизъюнкцию $n$ переменных, не может содержать функциональный оператор, вычисляющий тождественный нуль и выполняемый программой раньше, чем первый оператор остановки. Для удобства ссылок обозначим этом факт символом $\Delta$.

Предположим, что программа $P_{n}$, вычисляющая дизъюнкцию $n$ переменных, содержит функциональный оператор $p_{t}$, вычисляющий тождественный нуль и выполняемый программой раньше, чем первый оператор остановки $p_{j}: \operatorname{Stop}(A)$, то есть $t<j$. Из леммы 1 следует, что выходом такого оператора не может быть внутренняя переменная. Если выходом оператора является выходная переменная $Z$, то легко видеть, что вход $A(x ; j-1)$ первого оператора остановки должен существенно зависеть от всех $n$ переменных (здесь без ограничения общности полагаем, что между рассматриваемым функциональным оператором и первым оператором остановки нет ни одного функционального оператора, выходом которого является переменная $Z$ ). В противном случае значение дизъюнкции $n$ переменных будет равно нулю при единичном значении переменной, не являющейся существенным аргументом функции, вычисляемой на входе оператора остановки. Следовательно, $T\left(P_{n}\right) \geqslant n$. Получаем противоречие с верхней оценкой.

Пусть $P_{n}-$ минимальная программа, вычисляющая

$$
\vee_{n}=x_{1} \vee x_{2} \vee \ldots \vee x_{n}
$$


Отметим, что первым оператором любой программы, вычисляющей полностью определенную функцию, не может быть оператор остановки; перед оператором остановки необходим хотя бы один функциональный оператор, выходом которого является выходная переменная. Если первый оператор остановки программы $P_{n}$ является третьим оператором- $P_{n}$, то очевидно, что $T\left(P_{n}\right)>3$. Поэтому достаточно рассмотреть случай, когда в программе $P_{n}$ первый оператор остановки стоит на втором месте. Возможны два принципиально различных, с точностью до переименования переменных, случая для выбора первых двух операторов:

$$
\begin{array}{ll}
p_{1}: & Z=g\left(x_{1}, x_{2}\right), \quad Z=g\left(x_{1}, x_{2}\right), \\
p_{2}: & \operatorname{Stop}\left(x_{j}\right), \quad \operatorname{Stop}(Z),
\end{array}
$$

где $j \in\{1,2, \ldots, n\}$.

Рассмотрим первый случай. Без ограничения общности будем полагать $j=1$. В программе $P_{n}$ вместо переменной $x_{1}$ подставим нуль. Легко видеть, что новая программа $P_{n-1}$ вычисляет дизъюнкцию $n-1$ переменной и содержит по крайней мере на один оператор меньше, чем $P_{n}$, так как из $P_{n-1}$ можно удалить первый оператор остановки программы $P_{n}$. Следовательно,

$$
\sum_{x \in\{0,1\}^{n}, x_{1}=0} T_{P_{n}}\left(x_{1}, \ldots, x_{n}\right) \geqslant \sum_{x \in\{0,1\}^{n-1}}\left(T_{P_{n-1}}\left(x_{2}, \ldots, x_{n}\right)+1\right) .
$$

Так как

$$
\frac{1}{2^{n-1}} \sum_{x \in\{0,1\}^{n-1}} T_{P_{n-1}}\left(x_{2}, \ldots, x_{n}\right) \geqslant T\left(\vee_{n-1}\left(x_{2}, \ldots, x_{n}\right)\right),
$$

справедливы оценки

$$
\begin{aligned}
T\left(\vee_{n}\right) & =\frac{1}{2^{n}}\left(\sum_{x \in\{0,1\}^{n}, x_{1}=1} 2+\sum_{x \in\{0,1\}^{n}, x_{1}=0} T_{P_{n}}\left(x_{1}, \ldots, x_{n}\right)\right) \\
& \geqslant \frac{1}{2^{n}}\left(2 \cdot 2^{n-1}+\left(1+T\left(\vee_{n-1}\left(x_{2}, \ldots, x_{n}\right)\right)\right) 2^{n-1}\right) \\
& \geqslant 1+\frac{1}{2}+\frac{T\left(\vee_{n-1}\right)}{2}=\frac{3}{2}+\frac{T\left(\vee_{n-1}\right)}{2}
\end{aligned}
$$

Так как $T\left(\vee_{n-1}\right) \geqslant 2$ при $n \geqslant 4$, справедливы оценки

$$
T\left(\vee_{n-1}\right)=\frac{1}{2} T\left(\vee_{n-1}\right)+\frac{1}{2} T\left(\vee_{n-1}\right) \geqslant 1+\frac{1}{2} T\left(\vee_{n-1}\right)
$$

Поэтому из (1) следует, что

$$
T\left(\vee_{n}\right) \geqslant 2+\frac{1}{4} T\left(\vee_{n-1}\right)
$$

Рассмотрим второй случай. $\mathrm{B}$ программе $P_{n}$ вместо двух первых переменных подставим нули. Легко видеть, что новая программа $P_{n-2}$ вычисляет дизъюнкцию $n-2$ переменных и содержит по крайней мере на два оператора меньше, чем $P_{n}$, так как из $P_{n-2}$ можно удалить два первых оператора программы $P_{n}$. Первый оператор остановки, то есть оператор $p_{2}$, можно удалить, так как он не останавливает 
вычисления. Первый оператор программы $P_{n}$ вычисляет тождественный нуль. Из доказанного выше утверждения $\Delta$ следует, что после преобразования программы $P_{n-2}$ в приведенную программу, этот оператор не будет использован и поэтому может быть удален. Следовательно,

$$
\sum_{x \in\{0,1\}^{n}, x_{1}=x_{2}=0} T_{P_{n}}\left(x_{1}, \ldots, x_{n}\right) \geqslant \sum_{x \in\{0,1\}^{n-2}}\left(T_{P_{n-2}}\left(x_{3}, \ldots, x_{n}\right)+2\right) .
$$

Так как

$$
\frac{1}{2^{n-2}} \sum_{x \in\{0,1\}^{n-2}} T_{P_{n-2}}\left(x_{3}, \ldots, x_{n}\right) \geqslant T\left(\vee_{n-2}\left(x_{3}, \ldots, x_{n}\right)\right)
$$

справедливы оценки

$$
\begin{aligned}
T\left(\vee_{n}\right) & =\frac{1}{2^{n}}\left(\sum_{x \in\{0,1\}^{n}, x_{1} \vee x_{2}=1} 2+\sum_{x \in\{0,1\}^{n}, x_{1}=x_{2}=0} T_{P_{n}}\left(x_{1}, \ldots, x_{n}\right)\right) \\
& \geqslant \frac{1}{2^{n}}\left(2 \cdot 3 \cdot 2^{n-2}+\left(2+T\left(\vee_{n-2}\left(x_{3}, \ldots, x_{n}\right)\right)\right) 2^{n-2}\right) \\
& \geqslant \frac{3}{2}+\frac{1}{2}+\frac{T\left(\vee_{n-2}\right)}{4}=2+\frac{T\left(\vee_{n-2}\right)}{4} .
\end{aligned}
$$

Из (3) находим, что

$$
\begin{aligned}
T\left(\vee_{n}\right) & \geqslant 2+\frac{T\left(\vee_{n-2}\right)}{4} \geqslant 2+\frac{1}{2}+\frac{T\left(\vee_{n-4}\right)}{16} \geqslant \ldots \geqslant 2+\frac{1}{2}+\frac{1}{8}+\ldots \\
& \sim \frac{2}{1-1 / 4} \sim \frac{8}{3} .
\end{aligned}
$$

Такая же оценка аналогичным образом следует из (2).

Следовательно, $T\left(\vee_{n}\right) \sim 8 / 3$. Теорема доказана.

Теорема 2. $\Pi p u n \rightarrow \infty$

$$
T\left(x_{1} \& x_{2} \& \ldots \& x_{n}\right) \sim \frac{11}{3}
$$

Доказателъство. Верхнюю оценку среднего времени получим, оценив среднее время работы приводимой ниже программы $P_{\&}$, вычисляющей конъюнкцию $n$ переменных. Как и при доказательстве предыдущей теоремы, без ограничения общности 
полагаем, что $n-$ четное число.

$$
\begin{aligned}
p_{1}: & Y=\overline{x_{1} \& x_{2}}, \\
p_{2}: & Z=0, \\
p_{3}: & \operatorname{Stop}(Y), \\
p_{4}: & Y=\overline{x_{3} \& x_{4}}, \\
p_{5}: & \operatorname{Stop}(Y), \\
& \cdots \\
p_{j+1}: & Y=\overline{x_{j} \& x_{j+1}}, \\
p_{j+2}: & \operatorname{Stop}(Y), \\
& \cdots \\
p_{n-2}: & Y=\overline{x_{n-3} \& x_{n-2}}, \\
p_{n-1}: & \operatorname{Stop}(Y), \\
p_{n}: & Z=x_{n-1} \& x_{n} .
\end{aligned}
$$

Сравнивая программу $P_{\&}$ с программой $P_{\vee}$ из предыдущей теоремы, легко видим, что справедливо равенство $T\left(P_{\&}\right)=1+T\left(P_{V}\right)$. Следовательно,

$$
T\left(P_{\vee}\right) \leqslant 1+\frac{8}{3}=\frac{11}{3} \text {. }
$$

Верхняя оценка получена.

Для получения оценки снизу прежде всего отметим следующие утверждения, которые обозначим $\Delta_{1}$ и $\Delta_{2}$.

Утверждение $\Delta_{1}$ состоит в том, что среди первых $n-1$ операторов минимальной программы $P_{n}$, вычисляющей конъюнкцию $n$ переменных, нет оператора остановки, входом которого является выходная переменная.

Утверждение $\Delta_{2}$ состоит в том, что среди первых $n-2$ операторов минимальной программы $P_{n}$, вычисляющей конъюнкцию $n$ переменных, нет оператора остановки, входом которого является независимая переменная.

Докажем $\Delta_{1}$. Предположим, что такой оператор $p_{t}: \operatorname{Stop}(Z)$ в минимальной программе есть. Тогда на некотором наборе $\left(\sigma_{1}, \ldots, \sigma_{n}\right)$ этот оператор прекращает выполнение программы. Легко видеть, что выходная переменная $Z(x ; t-1)$ существенно зависит не более, чем от $n-1$ переменных. Без ограничения общности полагаем, что $Z(x ; t-1)$ не зависит от $x_{n}$. Тогда $Z(x ; t-1)$ равна единице на наборе $\left(\sigma_{1}, \ldots, \sigma_{n-1}, 0\right)$. Получаем противоречие.

Докажем $\Delta_{2}$. Предположим, что такой оператор $p_{t}: \operatorname{Stop}\left(x_{l}\right)$ в минимальной программе есть. Легко видеть, что выходная переменная $Z(x ; t-1)$ существенно зависит не более, чем от $n-2$ переменных. Без ограничения общности полагаем, что $Z(x ; t-1)$ не зависит от $x_{n-1}$ и $x_{n}$ и $n \neq l$. Тогда программа прекращает работу на наборе $x_{1}=x_{2}=\ldots=x_{n-1}=1$ и результат работы не зависит от $x_{n}$. Получаем противоречие.

Отметим также, что первым оператором любой программы, вычисляющей полностью определенную функцию, не может быть оператор остановки; перед оператором остановки необходим хотя бы один функциональный оператор, выходом которого является выходная переменная. 
Пусть $P_{n}$ - минимальная программа, вычисляющая функцию

$$
\&_{n}=x_{1} \& x_{2} \& \ldots \& x_{n}
$$

Если первый оператор остановки программы $P_{n}$ является четвертым оператором $P_{n}$, то очевидно, что $T\left(P_{n}\right)>4$. Поэтому достаточно рассмотреть случай, когда в программе $P_{n}$ первый оператор остановки стоит на втором или третьем месте.

Из $\Delta_{1}$ и $\Delta_{2}$ следует, что независимая и выходная переменные не могут быть входом оператора остановки, поэтому очевидно, что случай, когда в программе $P_{n}$ первый оператор остановки стоит на втором месте невозможен.

Рассмотрим случай, когда первый оператор остановки $s_{1}$ стоит на третьем месте. Из утверждений $\Delta_{1}$ и $\Delta_{2}$ легко следует, что возможны только три. принципиально различных, с точностью до переименования переменных, случая для выбора первых трех операторов:

$$
\begin{array}{rrr}
p_{1}: \quad Y=g\left(x_{1}, x_{2}\right) ; \quad Y=g\left(x_{1}, x_{2}\right) ; & Z=g\left(x_{1}, x_{2}\right), \\
p_{2}: & Z=f\left(Y, x_{3}\right) ; \quad Z=f\left(x_{3}, x_{4}\right) ; \quad Y=f\left(Z, x_{3}\right),
\end{array}
$$

$p_{3}: \quad \operatorname{Stop}(Y) ; \operatorname{Stop}(Y) ; \operatorname{Stop}(Y)$.

Так как вычисляемая первыми двумя операторами программы система функций $\{Y(x ; 2), Z(x ; 2)\}$ существенно зависит не более, чем от четырех переменных, при $n>4$ найдется переменная, например $x_{n}$, не являющаяся существенной для системы $\{Y(x ; 2), Z(x ; 2)\}$. На любом наборе с $x_{n}=0$ конъюнкция $n$ переменных равна нулю, поэтому очевидно, что

$$
Z\left(x_{1}, x_{2}, \ldots, x_{n} ; 2\right) \& Y\left(x_{1}, x_{2}, \ldots, x_{n} ; 2\right)=0
$$

при всех возможных значениях переменных $x_{1}, \ldots, x_{n-1}$ и $x_{n}=0$.

Далее рассмотрим два первых случая, когда функция $Y(x ; 2)$ существенно зависит только от двух переменных. Покажем, что при всех $\left(\sigma_{3}, \ldots, \sigma_{n}\right)$

$$
T_{P_{n}}\left(1,1, \sigma_{3}, \ldots, \sigma_{n}\right)>3
$$

то есть при $x_{1}=x_{2}=1$ первый оператор остановки программы $P_{n}$ не останавливает вычисления. Сделаем это методом от противного. Допустим, что найдется набор $\left(\sigma_{3}^{\prime}, \ldots, \sigma_{n}^{\prime}\right)$ такой, что

$$
T_{P_{n}}\left(1,1, \sigma_{3}^{\prime}, \ldots, \sigma_{n}^{\prime}\right)=3 \text {. }
$$

Следовательно, $Y(1,1 ; 2)=1$. Так как $Y(x ; 2)$ существенно зависит только от первых двух переменных, в силу (4)

$$
Z(1,1, \ldots, 1 ; 2) \& Y(1,1, \ldots, 1 ; 2)=0 .
$$

Поэтому $Z(1,1, \ldots, 1 ; 2)=0$. Получаем противоречие.

В программе $P_{n}$ вместо первых двух переменных подставим единицы и преобразуем ее в приведенную программу. Новая программа $P_{n-2}$ вычисляет конъюнкцию $n-2$ переменных и содержит по крайней мере на два оператора меньше, чем программа $P_{n}$. Из (5) следует, что первый оператор остановки программы $P_{n}$, то есть оператор $p_{3}$, можно удалить, так как после подстановки единиц вместо $x_{1}$ и $x_{2}$ он не останавливает вычисления. После этой подстановки первый оператор программы $P_{n}$ 
вычисляет тождественный нуль и его выход - внутренняя переменная. Из леммы 1 следует, что выход этого оператора не является входом никакого другого оператора $P_{n-2}$ и поэтому его можно удалить. Следовательно,

$$
\sum_{x \in\{0,1\}^{n}, x_{1}=x_{2}=1} T_{P_{n}}\left(x_{1}, \ldots, x_{n}\right) \geqslant \sum_{x \in\{0,1\}^{n-2}}\left(T_{P_{n-2}}\left(x_{3}, \ldots, x_{n}\right)+2\right) .
$$

Так как

$$
\frac{1}{2^{n-2}} \sum_{x \in\{0,1\}^{n-2}} T_{P_{n-2}}\left(x_{3}, \ldots, x_{n}\right) \geqslant T\left(\&_{n-2}\left(x_{3}, \ldots, x_{n}\right)\right),
$$

справедливы оценки

$$
\begin{aligned}
T\left(\&_{n}\right) & =\frac{1}{2^{n}}\left(\sum_{x \in\{0,1\}^{n}, x_{1} \& x_{2}=0} 3+\sum_{x \in\{0,1\}^{n}, x_{1}=x_{2}=1} T_{P_{n}}\left(x_{1}, \ldots, x_{n}\right)\right) \\
& \geqslant \frac{1}{2^{n}}\left(3 \cdot 3 \cdot 2^{n-2}+\left(2+T\left(\&_{n-2}\left(x_{3}, \ldots, x_{n}\right)\right)\right) 2^{n-2}\right) \\
& \geqslant \frac{9}{4}+\frac{2}{4}+\frac{T\left(\&_{n-2}\right)}{4}=\frac{11}{4}+\frac{T\left(\&_{n-2}\right)}{4} .
\end{aligned}
$$

Таким образом,

$$
\begin{aligned}
T\left(\&_{n}\right) & \geqslant \frac{11}{4}+\frac{T\left(\&_{n-2}\right)}{4} \\
& \geqslant \frac{11}{4}+\frac{11}{16}+\frac{T\left(\&_{n-4}\right)}{16} \geqslant \ldots \geqslant \frac{11}{4}\left(1+\frac{1}{4}+\frac{1}{16}+\ldots\right) \sim \frac{11}{4} \cdot \frac{1}{1-1 / 4} \sim \frac{11}{3} .
\end{aligned}
$$

Рассмотрим третий случай. Если $Y(x ; 2)$ существенно зависит только от двух переменных, то доказательство полностью аналогично доказательству в первых двух случаях. Поэтому далее полагаем, что $Y(x ; 2)$ существенно зависит от трех переменных, и следовательно, $Z(x ; 1)$ существенно зависит от двух переменных. Разложим $Y(x ; 2)$ по аргументу $Z$ :

$$
Y(x ; 2)=Z(x ; 1) f_{1}\left(x_{3}\right) \vee \bar{Z}(x ; 1) f_{2}\left(x_{3}\right) .
$$

Так как $Z(x ; 1)=Z(x ; 2)$, из (1) легко видеть, что

$$
0=Z(x ; 2) Y(x ; 2)=Z(x ; 2) f_{1}\left(x_{3}\right) \vee Z(x ; 2) \bar{Z}(x ; 2) f_{2}\left(x_{3}\right)=Z(x ; 2) f_{1}\left(x_{3}\right) .
$$

Следовательно, $f_{1}\left(x_{3}\right) \equiv 0$. Поэтому

$$
Y(x ; 2)=\bar{Z}(x ; 2) f_{2}\left(x_{3}\right) .
$$

Но тогда $Y\left(x_{1}, x_{2}, x_{3} ; 2\right)$ равна единице не более, чем на трех наборах значений переменных $x_{1}, x_{2}, x_{3}$. Поэтому первый оператор остановки прекращает вычисления не более, чем на $3 \cdot 2^{n-3}$ наборах значений переменных $x_{1}, \ldots, x_{n}$. На остальных $5 \cdot 2^{n-3}$ наборах должны выполниться еще хотя бы два оператора: оператор остановки и его аргумент - функциональный оператор (см. утверждения $\Delta_{1}$ и $\Delta_{2}$ ). Следовательно,

$$
T(P) \geqslant \frac{1}{2^{n}}\left(3 \cdot 3 \cdot 2^{n-3}+5 \cdot 5 \cdot 2^{n-3}\right)>4 .
$$

Теорема доказана. 
Из доказательства теорем 1, 2 легко видеть, что утверждения этих теорем справедливы также для дизъюнкций и конъюнкций вида

$$
\begin{aligned}
& x_{1}^{\sigma_{1}} \vee x_{2}^{\sigma_{2}} \vee \ldots \vee x_{n}^{\sigma_{n}} \\
& x_{1}^{\sigma_{1}} \& x_{2}^{\sigma_{2}} \& \ldots \& x_{n}^{\sigma_{n}}
\end{aligned}
$$

при произвольном наборе $\sigma_{1}, \ldots, \sigma_{n}$.

Теорема 3. Справедливо равенство

$$
T\left(x_{1} \oplus x_{2} \oplus \ldots \oplus x_{n}\right)=n-1
$$

Доказательство. Верхняя оценка достигается на программе без операторов остановки, то есть на обычной схеме из функциональных элементов, состоящей из $n-1$ элемента сложения по модулю два. Найдем нижнюю оценку. Если существует вычисляющая линейную функцию программа $P$, среднее время работы которой меньше, чем $n-1$, то очевидно, что эта программа содержит такой первый оператор остановки $p_{j}: \operatorname{Stop}(A)$, что $j<n-1$. В этом случае вход $A(x ; j-1)$ оператора остановки и выходная переменная $Z(x ; j-1)$ зависят вместе не более, чем от $n-1$ переменных. Без ограничения общности будем считать, что $A(x ; j-1)$ и $Z(x ; j-1)$ не зависят от $x_{n}$. Следовательно, найдется набор $\alpha_{1}, \ldots, \alpha_{n-1}$ такой, что

$$
A\left(\alpha_{1}, \ldots, \alpha_{n-1} ; j-1\right)=1 \text {. }
$$

Поэтому

$$
Z\left(\alpha_{1}, \ldots, \alpha_{n-1}, 1 ; j-1\right)=Z\left(\alpha_{1}, \ldots, \alpha_{n-1}, 0 ; j-1\right) .
$$

Получаем противоречие. Теорема доказана.

В заключение приведем ряд простых наблюдений, следующих из доказанных теорем. Первое связано с доказательством нижних оценок сложности булевых функций. В традиционных моделях вычислений, как правило, существует прямая связь между величиной доказываемой нижней оценки и сложностью доказательства - чем выше оценка, тем сложнее доказательство. В случае неветвящихся программ с условной остановкой ситуация иная - указанная связь сохраняется не для всех функций. Среднее время вычисления линейной функции на порядок больше среднего времени вычисления конъюнкции, в то время как доказательство соответствующей нижней оценки значительно проще.

Второе наблюдение связано с неоднократно отмеченным ранее существенным отличием линейной функции от остальных элементарных булевых функций: присутствие линейной функции в базисе вычисления обычно ведет либо к уменьшению сложности вычислений, либо к усложнению получения нижних оценок. В рассматриваемой модели это отличие появляется явно -- в большем среднем времени вычисления линейной функции.

Наконец отметим следующее. В $[2,4]$ установлено, что для почти всех булевых функций $n$ переменных среднее время вычисления их значений в постоянное число раз меньше, чем время, необходимое в худшем случае. Теоремы 1-3 дают примеры конкретных функций, для которых это не так. 


\section{Список литературы}

1. Лупанов О. Б., Асимптотические оченки сложности управляющих систем. Изд-во Моск. ун-та, Москва, 1984.

2. Чашкин А. В., О среднем времени вычисления значений булевых функций. Дискретный анализ и исследование операчий (1997) 4, №1, 60-78.

3. Чашкин А. В., О вычислении булевых функций вероятностными программами. Дискретный анализ и исследование операчий (1997) 4, №3, 49-68.

4. Чашкин А. В., О среднем времени вычисления значений булевых операторов. Дискретный анализ и исследование операций (1998) 5, № 1, 88-103.

5. Чашкин А. В., О среднем времени вычисления полиномиально сводимых булевых функций. Вестних Мосх. ун-та. Серия 1. Мат. Мех. (1998) № 1, 68-71.

6. Чашкин А. В., О реализации линейных булевых операторов неветвящимися программами с условной остановкой. Дискретная математиха (1999) 11, № 1, 146-150.

Статья поступила 14.07.2000. 